\title{
A novel method to calculate a near field of widely divergent laser beams
}

\author{
Emilia Pruszyńska-Karbownik ${ }^{1}$ Kazimierz Regiński ${ }^{1} \cdot$ \\ Maciej Bugajski ${ }^{1}$
}

Received: 5 November 2015/ Accepted: 15 April 2016/Published online: 28 April 2016

(C) The Author(s) 2016. This article is published with open access at Springerlink.com

\begin{abstract}
A new method to calculate the near field of widely divergent laser beams based on a given far field is proposed. It is assumed that the field of radiation consists of several transversal modes and the far field is determined by the Huygens principle. The idea consists in employing the least squares method to find a minimum of the difference between the measured far field and the far field calculated under the assumption that is consists of several transversal modes with assumed weights. To find this minimum the Levenberg-Marquardt algorithm is used. Thus the problem is dealt beyond the paraxial approximation. The method is illustrated by its results for experimental mid-infrared quantum cascade laser beams and for the beams obtained by simulations.
\end{abstract}

Keywords Near field · Far field · Quantum cascade laser · Non-Gaussian beam

\section{Introduction}

Semiconductor lasers are devices of complex structure contained in compact sizes. Physical processes inside the laser resonators are not well understood yet, inter alia because of the experimental difficulties. These internal processes affect fields distributions on the laser mirror, and thereby the optical beam. Therefore the beam shape is a symptom of the laser state and could be of use to semiconductor laser diagnostics.

This article is part of the Topical Collection on Advances in the Science of Light.

Guest Edited by Jelena Radovanovic, Milutin Stepic, Mikhail Sumetsky, Mauro Pereira and Dragan Indjin.

Emilia Pruszyńska-Karbownik

ekarb@ite.waw.pl

1 Institute of Electron Technology, al. Lotników 32/46, 03-668 Warsaw, Poland 
The transformation between the field distribution on the mirror (near field) and the field distribution in space (far field) is commonly performed based on paraxial approximation (Hodgson and Weber 2005). This approach is eligible for lasers that have low-divergent beams but not for edge-emitting semiconductor lasers whose beams have divergence of tens of degrees. Nonetheless, the approximation is also commonly used for analyzing the widely divergent beams, mainly because of convenience of invariant parameter $M^{2}$ which results from the approximation (Hodgson and Weber 2005). However, the use of $M^{2}$ is criticized for its imprecision and sensitivity to measurement errors (Siegman 1993; Jones and Scott 1994).

In addition, the transformation between the near- and far field is usually performed only in one direction: from the near- to far field. The reverse transformation cannot be done analytically without approximations. Mathematically it is an ill-posed problem (Regińska and Regiński 2015). However, the regularization problem is beyond the scope of this paper. We present the method of the far- to near field transformation which is, according to our knowledge, the first analysis method of non-Gaussian beams that does not use the paraxial approximation.

\section{Methods}

\subsection{Numerical method}

According to the effective index method a 2D waveguide can be treated as two mutually perpendicular one-dimensional (1D) waveguides. We assume that in one direction the waveguide is homogeneous and surrounded by metal. In the QCLs such a condition is fulfilled in the slow axis. In this case the field $E_{m}(x)$ of $m$ th transversal mode on the resonator mirror can be expressed by Szymański et al. (2013)

$$
E_{m}(x)=E_{m}^{\max } \sin \frac{(m+1) \pi x}{d}
$$

where $E_{m 0}^{\max }$ is maximal amplitude of the mode, and $d$ is the waveguide width.

The field distribution $E(x)$ of interfering transversal modes with amplitudes $E_{i}(x), E_{j}(x), E_{k}(x), \ldots$ and phase differences $\phi_{i j}, \phi_{i k}, \ldots$ is given by

$$
E(x)=E_{i}(x)+E_{j}(x) \exp i \phi_{i j}+E_{k}(x) \exp i \phi_{i k}+\ldots
$$

Rejecting the paraxial approximations, we transform the near- to far field by using Huygens principle. Having the field amplitude distribution on the laser mirror $E(x)$, we compute the field amplitude $E(\theta)$ in a certain $\mathrm{P}$ by summation of spherical waves coming from different points $x$ with the wavelength $\lambda$ :

$$
E(\theta)=C \int_{-\infty}^{\infty} \frac{E(x)}{r(x, \theta)} \exp -i \frac{2 \pi}{\lambda} r(x, \theta) d x,
$$

where $C$ is a proportionality factor. The distance $r(x, \theta)$ is

$$
r(x, \theta)=\sqrt{R^{2}-2 R x \sin \theta+x^{2}},
$$

where $R$ is the distance between the point $\mathrm{P}$ and the center of the mirror, and $\theta$ is the angle between the line connecting these points and the direction of light propagation. The power 
of light $P_{\operatorname{sim}}(\theta)$ is a linear function of squared module of the field amplitude $E(\theta)$ calculated from formula 3 .

To calculate the near field based on a given far field $P_{\text {giv }}$, we assume that the near field consists of 1 to 4 first transversal modes ( $m=0,1,2$ or 3 ) and its distribution is given by the formulae 1 and 2. For $m>0$ we consider the maximum amplitudes $E_{m}^{\max }$ and the phase differences $\phi_{0 m}$ as unknown parameters, while $E_{0}^{\max }$ is eliminated by normalization of the distributions. In the case of multiple modes we assume a certain $\lambda$, and for a single mode $\lambda$ is an unkown parameter. Thus, the vector of the sought parameters is $\beta=\left[E_{1}^{\max }, \phi_{01}, \ldots\right]$ for multiple modes, or $\beta=[\lambda]$ for a single mode. The essence of the method is the minimalization of the functional

$$
S(\beta)=\sum_{i=1}^{m}\left(\frac{P_{g i v}\left(\theta_{i}\right)}{\sum_{i=1}^{m} P_{g i v}\left(\theta_{i}\right)}-\frac{P_{s i m}\left(\theta_{i}, \beta\right)}{\sum_{i=1}^{m} P_{s i m}\left(\theta_{i}, \beta\right)}\right)^{2}
$$

(in the sence of the least squares method). To find this minimum, we use the LevenbergMarquardt algorithm (Wolberg 2006), which gives us values of the unknown parameters, and-as the final result — the near field.

\subsection{Far-field simulations}

We perfomed far-field simulations by using the above model with assumption of one or two first transversal modes with optional noise or tilt of the laser mirror. We assumed $\lambda=9.5 \mu \mathrm{m}, \phi_{01}=0.5 \pi$ and $d=15 \mu \mathrm{m}$. The noise was introduced by multiplying each power value by a random number with normal distribution. The tilt was introduced by adding a constant value to the angle.

\subsection{Experiment}

We measured the far fields for quantum cascade lasers (QCLs) of three epitaxial structures. $\mathrm{GaAs} / \mathrm{GaAs}$ laser has an active region made of $\mathrm{GaAs}$ and $\mathrm{Al}_{0.45} \mathrm{GaAs}$ and it was grown on GaAs substrate. InGaAs/GaAs laser has $\mathrm{In}_{0.03} \mathrm{GaAs} / \mathrm{Al}_{0.45} \mathrm{GaAs}$ active region and was grown on GaAs too. InGaAs/InP laser has an active region made of InGaAs and InAlAs lattice matched to InP substrate. Detailed information about the technology can be found in Kosiel et al. (2009), Karbownik et al. (2014), Bugajski et al. (2014).

All measurements were provided by using a goniometric profilometer (PruszyńskaKarbownik et al. 2013). On the basis of two-dimensional (2D) power distributions, we calculated distributions in direction parallel to the epitaxial layers (slow axis) by summation over perpendicular direction (fast axis). This gives a result identical with that measured by using a moving slit.

\section{Results}

Exemplary results of our calculation method for experimental far-field distributions are shown in Figs. 1 and 2. Figure 1 presents results for laser beam which is similar to Gaussian beam (quasi-Gaussian beam) and Fig. 2 presents results for non-Gaussian laser beam. In both the cases we used the multi-mode calculations, but in the case of quasiGaussian beam the single-mode calculations give the same near-field distribution. It shows the existence of a single transverse mode in this beam. 

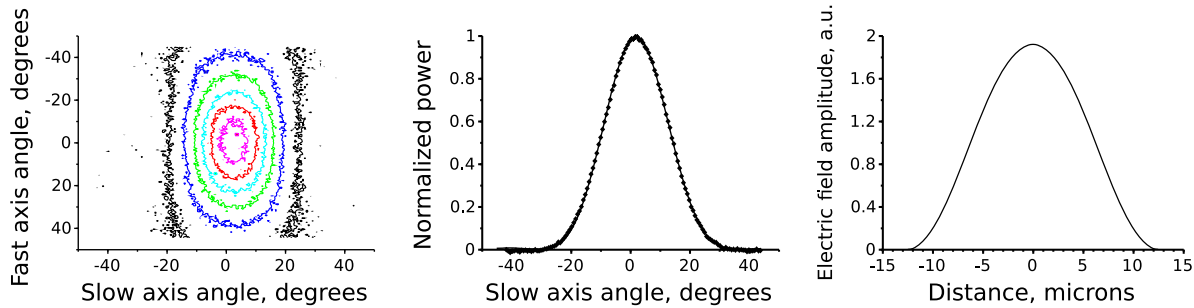

Fig. 1 2D far-field distribution (on the left), 1D far-field distribution for the slow axis (in the middle) and near-field distribution calculated on its basis for InGaAs/GaAs QCL emitting quasi-Gaussian beam (on the right)
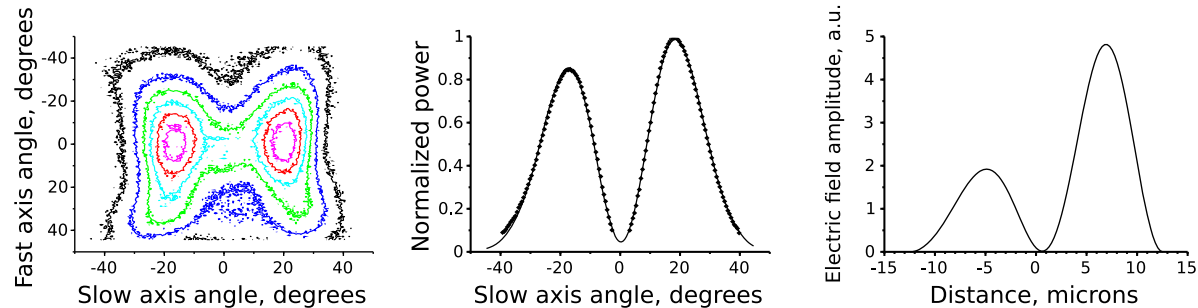

Fig. 2 2D far-field distribution (on the left), 1D far-field distribution for the slow axis (in the middle) and near-field distribution calculated on its basis for InGaAs/GaAs QCL emitting non-Gaussian beam (on the right)

Table 1 Comparison of wavelengths measured by using Fourier transform infra-red spectrometer (FTIR) and estimated from far-field measurements for different quantum cascade laser structures

\begin{tabular}{lll}
\hline Structure & Central wavelength (FTIR) & $\begin{array}{l}\text { Estimated wavelength } \\
\text { (from far-field) }\end{array}$ \\
\hline GaAs/GaAs & $9.6 \mu \mathrm{m}(238 \mathrm{~K})$ (Bugajski et al. 2014) & $9.8 \mu \mathrm{m}(293 \mathrm{~K})$ \\
$\mathrm{InGaAs} / \mathrm{GaAs}$ & $9.48 \mu \mathrm{m}(300 \mathrm{~K})$ (Pierściński et al. 2014) & $9.50 \mu \mathrm{m}(293 \mathrm{~K})$ \\
$\mathrm{InGaAs} / \mathrm{InP}$ & $9.2 \mu \mathrm{m}(283 \mathrm{~K})$ (Bugajski et al. 2014) & $9.3 \mu \mathrm{m}(293 \mathrm{~K})$
\end{tabular}

The ambient temperatures of the measurements are given in the brackets

Table 1 presents wavelengths estimated by the single-mode calculations for various QCL structures and wavelengths obtained by using Fourier transform infra-red spectrometer (FTIR) for lasers made with the same technology. In all cases the estimated wavelength is larger than that obtained by FTIR. The difference is from 0.02 to $0.2 \mu \mathrm{m}$. It should be noted that in the last case the ambient temperatures for both the experiments differed by $55 \mathrm{~K}$, which increased the difference of the wavelengths.

For testing the multi-mode calculations, we checked how the measurement accuracy affects the results. We checked that the optical power distributions in one spatial point are normal with the standard derivation $\sigma \leq 2 \%$. Figure 3 presents the result of the simulation of the fundamental mode with added the noise with $\sigma=2 \%$. The single-mode calculations resulted the wavelength which is lower from the proper one by $0.11 \mu \mathrm{m}$. The two-mode calculations gave the near field close to the original one. The distributions obtained for more modes are noticeably different. 

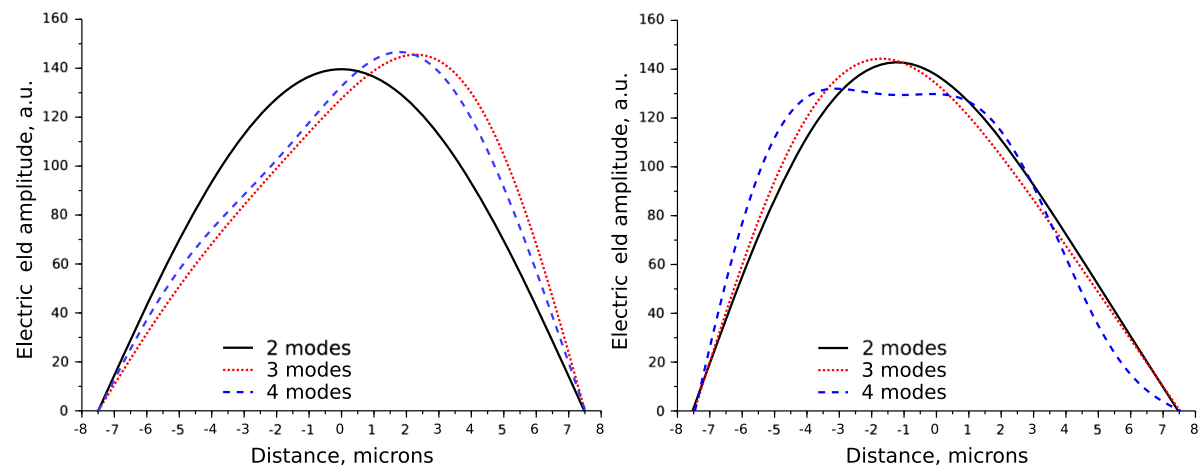

Fig. 3 Near fields calculated on basis of fundamental mode far field with noise of $\sigma=2 \%$ (on the left) and on basis of fundamental mode far field with the mirror tilt of $5^{\circ}$ (on the right)
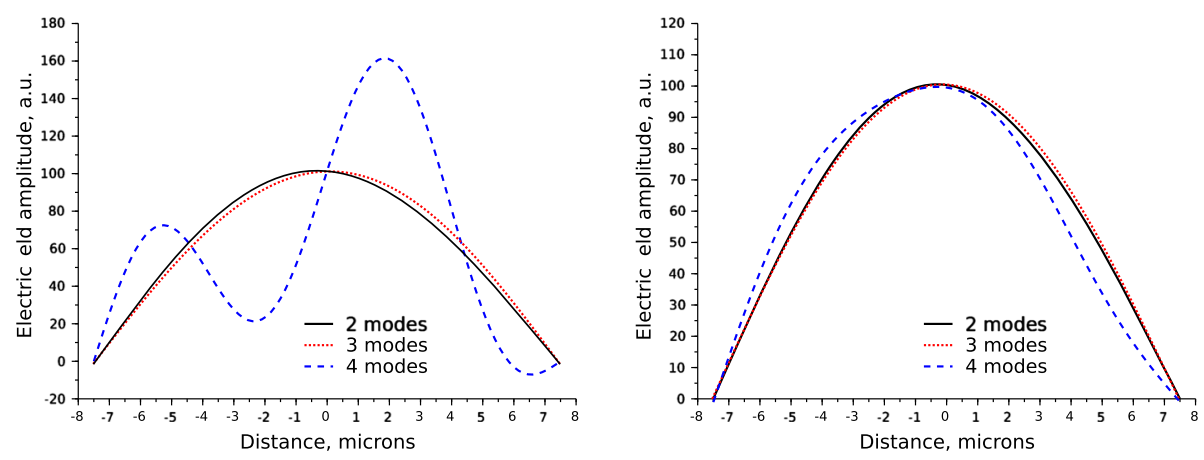

Fig. 4 Near field obtained by calculations for the simulation results that was conducted for 15 - $\mu$ m-width active region emitting two lowest order mode. $\phi_{01}=0.5 \pi, \lambda=9.5 \mu \mathrm{m}$ and $\sigma=2 \%$. The calculations were made on assumption of 2-4 lowest order modes. The initial near-field function for least square method is equal share of all the modes and $\phi_{01}=0$ (on the left) or $\phi_{01}=0.5 \pi$ (on the right)

A tilt of the laser mirror relative to the axis of the set-up may be another source of the experimental errors. Figure 3 presents results of the beam with the mirror tilt of $5^{\circ}$. The tilt does not influence on the estimated wavelength. However, when we assume more than one modes, the received near fields evidently differ from the original symmetrical one.

The non-linear least squares method is sensitive to initial parameters. The previously presented results do not depend on the choice of the initial parameters. We perform simulation of two interfering modes to examine this aspect. Figure 4 presents the near fields for different parameters. The results show that the calculation method is insensitive to the initial mode amplitudes, but can be sensitive to the initial phase differences, especially for many modes.

\section{Discussion}

Using the least squares method, we made transformation from the far- to near field without using the paraxial approximation. The single-mode calculations resulted in wavelengths which coincide with FTIR results. We interpreted this fact as a proof of the method 
correctness. We used sine functions as the assumed near fields. In our opinion it is sufficient for such an analysis, but similar analysis can also be done by using other laser waveguide models, including 2D ones.

Our results show that the far-field measurement accuracy has a large impact on the correctness of the calculations. Some weakness of this approach is sensitivity to the initial functions in the least squares method, especially for large number of assumed modes. Taking this into account, we recommend using the minimal number of the modes that gives reasonable results.

For a final evaluation of the method correctness, the obtained experimental results should be compared with measured near field distributions for the same laser structures.

Acknowledgments We would like to thank K. Kosiel, P. Gutowski, A. Szerling, P. Karbownik and A. Trajnerowicz for making their quantum cascade lasers available for us. The authors acknowledge support from MPNS COST ACTION MP1204.

Open Access This article is distributed under the terms of the Creative Commons Attribution 4.0 International License (http://creativecommons.org/licenses/by/4.0/), which permits unrestricted use, distribution, and reproduction in any medium, provided you give appropriate credit to the original author(s) and the source, provide a link to the Creative Commons license, and indicate if changes were made.

\section{References}

Bugajski, M., et al.: Mid-IR quantum cascade lasers: device technology and non-equilibrium Green's function modeling of electro-optical characteristics. Phys. Status Solidi. 251, 1144-1157 (2014)

Hodgson, N., Weber, H.: Laser Resonators and Beam Propagation. Springer, Berlin (2005)

Jones, R.D., Scott, T.R.: Error propagation in laser beam spatial parameters. Opt. Quantum Electron. 26, 25-34 (1994)

Karbownik, P., et al.: Direct AuAu bonding technology for high performance GaAs/AlGaAs quantum cascade lasers. Opt. Quantum Electron. 47(4), 893-899 (2014)

Kosiel, K., et al.: Molecular beam epitaxy growth for quantum cascade lasers. Acta Physica Polonica A 116, 806-813 (2009)

Pierściński, K., et al.: High performance room temperature InGaAs/AlGaAs/GaAs quantum cascade lasers. Mid Infrared Optoelectronics Materials and Devices XII, Montpellier, France, 5-9 Oct 2014

Pruszyńska-Karbownik, E. et al.: Analysis of the spatial distribution of radiation emitted by MIR quantum cascade lasers. In: Proceedings of SPIE, 8702, 87020E1-870210E8 (2013)

Regińska, T., Regiński, K.: Regularization strategy for determining laser beam quality parameters. J. Inverse Ill-Posed Probl. 23, 657-671 (2015)

Siegman, A.: Defining, measuring, and optimizing laser beam quality. Proc. SPIE 1868, 2-12 (1993)

Szymański, M. et al.: Calculation of beam divergence of a quantum cascade laser by effective index method. In: Proceedings of SPIE, 8702, 87020G1-87020G6 (2013)

Wolberg, J.: Data Analysis Using the Method of Least Squares. Springer, Berlin (2006) 